\title{
ERRATUM
}

\section{Erratum to: Real time qPCR expression analysis of some stress related genes in leaf tissue of Pyrus communis cv. Conférence after infection with Erwinia amylovora}

\author{
K. Vrancken • H. Schoofs • T. Deckers • \\ R. Valcke
}

Published online: 3 July 2012

(C) Springer-Verlag 2012

Erratum to: Trees (2012) 26:67-73

DOI 10.1007/s00468-011-0666-3

In the original version of Table 1, the primer sequences for APXI are wrong. The correct left primer is CCCCCA TAACCTACAACC and the right primer is GACCTGT GAGCTTCCTCCT. The corrected table is reproduced on the following page.

The online version of the original article can be found under doi:10.1007/s00468-011-0666-3.

K. Vrancken $(\bowtie) \cdot R$. Valcke

Molecular and Physical Plant Physiology, Hasselt University,

Agoralaan Building D, 3590 Diepenbeek, Belgium

e-mail: kristof.vrancken@uhasselt.be

H. Schoofs - T. Deckers

Pomology Department, PC Fruit Research Station,

Fruittuinweg 1, 3800 Sint-Truiden, Belgium 
Table 1 Used primers for real time qPCR

\begin{tabular}{|c|c|c|c|}
\hline Genes & Accession Nr. & Left primer & Right primer \\
\hline FSD1 & Malus_v4_Contig16258 & GCCGTTAACCCCCTTATTTG & CTGCTTCCCATGATACGAGTT \\
\hline FSD2 & Malus_v4_Contig18456 & AGAATATCACTGGGGGAAGCA & GCGCTGCCTGGTTGAAAG \\
\hline FSD3 & Malus_v4_Contig5924 & GTCCTTCAGCAGATAGAAAAGG & AAGCCAAACCCAGCCAGA \\
\hline$M S D 1$ & Malus_v4_Contig8113 & CTTTGGTTCCATTGCTTGGT & CGGCTCAAGTGCTTTCTTTC \\
\hline CSD1 & Malus_v4_Contig18911 & TGGTTAAGGGTGTTGCTGTTC & GGGCACCATGCTCTTTTC \\
\hline$C S D 2$ & Malus_v4_Contig18796 & CATATCCACAGGACCACATTTC & AAGTTCGTGTCCACCCTTTC \\
\hline CSD3 & Malus_v4_Contig6927 & CAGATGGAGTTGCTGAGGTTT & АCTCTTGCTCCTGCGTTCC \\
\hline$A P X 1$ & CN894028.1 & СССССАТААССТАСААСС & GACCTGTGAGCTTCCTCCT \\
\hline$A P X 2$ & Malus_v4_Contig22437 & GGCGGTAGAAAAATGCAAGA & CTCCAAAAGCCTAACCACGA \\
\hline$A P X 3$ & Malus_v4_Contig8946 & TGGGTAGAGCACATCCAGAAA & TCAACATAGCGGCGGAAC \\
\hline$A P X 4$ & Malus_v4_Contig23178 & GCTGTTATGTCTGCATTCTTGG & CGGCTGCGTTGGTATTTCT \\
\hline$A P X 5$ & Malus_v4_Contig8946 & GTTCCCTTCCATCCTTCGTT & GTTTCGGCGTTGTATGTTCC \\
\hline CAT1 & Malus_v4_Contig4354 & CCGTGATGCAATGAAAATCC & CATCGAATAGGAAGGCGAAC \\
\hline CAT3 & Malus_v4_Contig3679 & TAATCACAGCCACGCTACCA & TTCAGTACCAAACGGCCAAC \\
\hline Ef $1-a$ & DQ341381.1 & AATCGCCTTTGTTCCCATC & GCACAGTTCCAATACCACCA \\
\hline Ef 4-a & AY347787.1 & ATCAGGCTCATCCCGTGT & AGCAACACССТTCCTTCC \\
\hline Clathrin & CN865508.1 & CGCTCTCCATTCTCTTCCA & CCTTCCCGGTTACATCACA \\
\hline
\end{tabular}

